\title{
Controller synthesis for interconnected systems using parametric assume-guarantee contracts
}

\author{
Mohammad Al Khatib and Majid Zamani
}

\begin{abstract}
In this paper we synthesize controllers for interconnected systems to enforce parametric assume-guarantee contracts (AGC), which encode behaviors of the systems in some parameter domains. In our approach to solve the design problem, we synthesize a controller for each component of the system so that it satisfies its own parametric AGC separately, and then define a mapping that generates the sequence of parameters for which the corresponding contracts are satisfied after interconnecting the components. Then if a small-gain like condition on the sequence of parameters hold, a parametric AGC is declared for the interconnected system. The classical small-gain theorem on bounded input bounded output (BIBO) stability is recovered by the obtained results showing the relation between the assume-guarantee reasoning and the smallgain approach. We also provide an example of a large-scale transportation system to illustrate our results.
\end{abstract}

\section{INTRODUCTION}

The computational complexity of synthesizing controllers monolithically for interconnected system (power networks, transportation systems, multi-robot systems, etc.) is often exponential in the number of interacting components. One technique to address this state-explosion problem is by using assume-guarantee contracts. AGCs enable a "divide-andconquer" approach for designing controllers compositionally, by designing the systems' components separately while making assumptions on each component's environment [1], [2]. In this context we consider a notion of parametric AGCs [3], encoding behaviors of a system in a parameter domain. Indeed, parametrization of contracts allows for tighter guarantees on the system's behavior since the system must satisfy only the guarantees whose assumptions are triggered. On the other hand, a contract with a different form, e.g. fixed AGCs [4], [5], has a coarse guarantee since it is designed based on the worst case assumption on the system's environment.

Here, we study the parametric AGC design problem: given an interconnected system composed of $N \geq 2$ components and $N$ parametric AGCs for each component, design a controller so that the interconnected system satisfies a global property. In our approach to solve the parametric AGC synthesis problem, we design a controller for each system's component separately to satisfy its own contract and then impose conditions on the contracts' guarantees and

This work was supported in part by the H2020 ERC Starting Grant AutoCPS (grant agreement No. 804639).

Mohammad Al Khatib is with the department of Electrical and Computer Engineering, Technical University of Munich, Germany. mohammad.al-khatib@tum.de

Majid Zamani is with the Computer Science Department, University of Colorado Boulder, USA. M. Zamani is with the Computer Science Department, Ludwig Maximilian University of Munich, Germany. majid.zamaniecolorado.edu assumptions. We also define a mapping that generates the sequence of parameters for which the parametric AGCs are satisfied after interconnecting the system's components. If this sequence of parameters converges to a limit point, a new parametric AGC is declared for the interconnected system.

The classical small-gain theorem on bounded input bounded output (BIBO) stability is recovered by the obtained results here showing the relation between the assumeguarantee reasoning and the classical trajectory-based smallgain theorems [6]. In addition to BIBO stability, more complex specifications for designing an interconnected system, such as a fragment of linear temporal logic (LTL) specifications, can be embedded in the framework of parametric AGCs here. Finally, we demonstrate our results by synthesizing a controller to enforce a temporal logic specification on a large-scale transportation system.

Various compositional approaches are developed in the literature to address the state-explosion problem when studying interconnected system. In the traditional control theory literature which is concerned mainly with stability properties, compositional methods in the form of small-gain theorems (SGTs) are established in [7], [6] for continuous-time systems and in [8] for discrete-time systems. Those SGTs mainly provide a general condition guaranteeing stability of a system described as an interconnection of stable subsystems. In the formal verification and symbolic controller synthesis literature, where the desired properties on the system become much more complex (e.g. temporal logic properties [9]), several compositional results are presented. See, e.g., [10], [11], [12], [13], [14], [15] for abstraction-based controller synthesis approaches and [16], [17], [4], [5], [18], [19] for methods relying on assume-guarantee reasoning. Our approach is mostly related to the work in [3], [20], where the authors consider verification of interconnected systems via parametric AGC, instead of fixed AGCs as in [4], [5]. This work uses the former approach to synthesize controllers for interconnected systems, composed of an arbitrary given number of components, using parametric AGCs.

This manuscript is organized as the following: after the introduction, the parametric AGC design problem is formulated in Section II. The problem's solution is presented in Section III where the main result is established in Theorem 1. Section V shows how the classical small-gain theorem can be recovered using the main of this paper. An example on a transportation network illustrates the effectiveness of our approach in Section VI before concluding our work. We note that proofs are ommited due to space limitations. 


\section{Notations}

Let $\mathbb{R}, \mathbb{R}_{0}^{+}, \mathbb{R}^{+}, \mathbb{N}, \mathbb{N}^{+}$denote the sets of reals, nonnegative reals, positive reals, non-negative integers and positive integers, respectively. For $I \subseteq \mathbb{R}_{0}^{+}$, let $\mathbb{N}_{I}=\mathbb{N} \cap I$. For $N \in \mathbb{N}$ we denote by $\bar{N}(i)$ the set $\mathbb{N}_{[1, N]} \backslash\{i\}$. For a set $\mathcal{S}$, we denote the set of all subsets of $\mathcal{S}$ by $2^{\mathcal{S}}$. We denote by $\operatorname{cl}(\mathcal{S})$ the closure of the set $\mathcal{S}$. We denote by $M=\operatorname{diag}\left(a_{1}, \ldots, a_{N}\right)$ a matrix with diagonal elements $M_{i i}=a_{i}, i \in \mathbb{N}_{[1, N]}$, and zero off-diagonal elements.

\section{PRoblem Formulation}

In this paper a dynamical system is defined as a relation between internal input signals, control input signal, external input signals, and output signals.

Definition 1: A Dynamical $\Sigma\left(\mathcal{U}_{e}[\cdot], \mathcal{U}_{c}[\cdot], \mathcal{U}_{f}[\cdot], \mathcal{Y}[\cdot], \mathcal{K}\right)$ is a relation:

system

$$
\Sigma\left(\mathcal{U}_{e}[\cdot], \mathcal{U}_{c}[\cdot], \mathcal{U}_{f}[\cdot], \mathcal{Y}[\cdot], \mathcal{K}\right) \subseteq \mathcal{U}_{e}[\cdot] \times \mathcal{U}_{c}[\cdot] \times \mathcal{U}_{f}[\cdot] \times \mathcal{Y}[\cdot],
$$

where $\mathcal{U}_{e}[\cdot], \mathcal{U}_{c}[\cdot], \mathcal{U}_{f}[\cdot]$, and $\mathcal{Y}[\cdot]$ are the external input, control input, internal input, and output sets of signals respectively. Moreover, $\mathcal{K}: \mathcal{Y}[\cdot] \rightarrow \mathcal{U}_{c}[\cdot]$ is a control map and any $\left(u_{e}[\cdot], u_{c}[\cdot], u_{f}[\cdot], y[\cdot]\right) \in \Sigma\left(\mathcal{U}_{e}[\cdot], \mathcal{U}_{c}[\cdot], \mathcal{U}_{f}[\cdot], \mathcal{Y}[\cdot], \mathcal{K}\right)$ satisfies $u_{c}[\cdot]=\mathcal{K}(y[\cdot])$.

We assume that any input $\left(u_{e}[\cdot], u_{c}[\cdot], u_{f}[\cdot]\right) \in \mathcal{U}_{e}[\cdot] \times \mathcal{U}_{c}[\cdot] \times$ $\mathcal{U}_{f}[\cdot]$ is paired with at least one $y[\cdot] \in \mathcal{Y}[\cdot]$ using the relation $\Sigma\left(\mathcal{U}_{e}[\cdot], \mathcal{U}_{c}[\cdot], \mathcal{U}_{f}[\cdot], \mathcal{Y}[\cdot], \mathcal{K}\right)$. Such $y[\cdot]$ is unique if (1) is deterministic, and we write $y[\cdot]=\Sigma\left(u_{e}[\cdot], \mathcal{K}(y[\cdot]), u_{f}[\cdot]\right)$, otherwise $y[\cdot]$ is not unique where in this case we say that $y[\cdot] \in \Sigma\left(u_{e}[\cdot], \mathcal{K}(y[\cdot]), u_{f}[\cdot]\right)$. If a dynamical system does not have internal inputs then Definition 1 reduces to $\Sigma\left(\mathcal{U}_{e}[\cdot], \mathcal{U}_{c}[\cdot], \mathcal{Y}[\cdot], \mathcal{K}\right)$. We use the latter definition when we define the interconnected system in Section II-A. Note that in some cases and for the sake of brevity we just use the notion $\Sigma$ for a dynamical system.

\section{A. Interconnected system}

The notion of interconnection is given here similar to [21, Definition 3.1]:

Definition 2: Given an output set $\mathcal{Y}[\cdot]$ and an internal input set $\mathcal{U}_{f}[\cdot]$, an interconnection $\mathcal{I}$ is a tuple $\mathcal{I}=$ $\left(\mathcal{Y}[\cdot], \mathcal{U}_{f}[\cdot], \mathcal{G}\right)$, where $\mathcal{G}: \mathcal{Y}[\cdot] \mapsto \mathcal{U}_{f}[\cdot]$ is a set-valued map that maps output signals to internal input signals.

Now we formally define an interconnected system $\Sigma$.

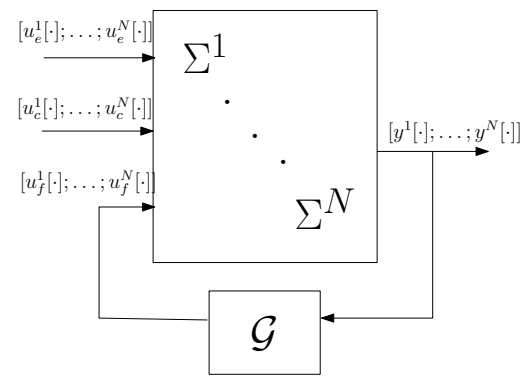

Fig. 1: The interconnected system $\mathcal{I}\left(\Sigma^{1}, \ldots, \Sigma^{N}\right)$.
Definition 3: Consider $N \in \mathbb{N}^{+}$subsystems $\Sigma^{i}\left(\mathcal{U}_{e}^{i}[\cdot], \mathcal{U}_{c}^{i}[\cdot], \mathcal{U}_{f}^{i}[\cdot], \mathcal{Y}^{i}[\cdot], \mathcal{K}^{i}\right), \quad i \quad \in \quad \mathbb{N}_{[1, N]}, \quad$ and $\quad$ an interconnection

$$
\mathcal{I}=\left(\prod_{i=1}^{N} \mathcal{Y}^{i}[\cdot], \prod_{i=1}^{N} \mathcal{U}_{f}^{i}[\cdot], \mathcal{G}\right)
$$

defining the coupling between these subsystems. We define an interconnected system $\mathcal{I}\left(\Sigma^{1}, \ldots, \Sigma^{N}\right)$ as a relation $\Sigma\left(\mathcal{U}_{e}[\cdot], \mathcal{U}_{c}[\cdot], \mathcal{Y}[\cdot], \mathcal{K}\right)$, with $\mathcal{U}_{e}[\cdot]=\prod_{i=1}^{N} \mathcal{U}_{e}^{i}[\cdot], \mathcal{U}_{c}[\cdot]=$ $\prod_{i=1}^{N} \mathcal{U}_{c}^{i}[\cdot], \quad \mathcal{Y}[\cdot]=\prod_{i=1}^{N} \mathcal{Y}^{i}[\cdot]$, where internal inputs of $\Sigma^{1}, \ldots, \Sigma^{N}$ are constrained as

$$
\left[u_{f}^{1}[\cdot] ; \ldots ; u_{f}^{N}[\cdot]\right] \in \mathcal{G}\left(\left[y^{1}[\cdot] ; \ldots ; y^{N}[\cdot]\right]\right),
$$

and control inputs are defined by

$$
\begin{aligned}
{\left[u_{c}^{1}[\cdot] ; \ldots ; u_{c}^{N}[\cdot]\right] } & =\mathcal{K}\left(\left[y^{1}[\cdot] ; \ldots ; y^{N}[\cdot]\right]\right), \\
& =\left[\mathcal{K}^{1}\left(y^{1}[\cdot]\right) ; \ldots ; \mathcal{K}^{N}\left(y^{N}[\cdot]\right)\right] .
\end{aligned}
$$

\section{B. Assume-guarantee contracts}

In the sequel, a specification $\Phi$ over the set $\mathcal{Z}[\cdot]$ describes a set of desirable input-output behaviors, and in the set point of view it satisfies the set inclusion $\Phi \subseteq \mathcal{Z}[\cdot]$. For example an input (or output) specification $\Phi$, for system $\Sigma\left(\mathcal{U}_{e}[\cdot], \mathcal{U}_{c}[\cdot], \mathcal{Y}[\cdot], \mathcal{K}\right)$, satisfies $\Phi \subseteq \mathcal{U}_{e}[\cdot]$ (or $\left.\Phi \subseteq \mathcal{Y}[\cdot]\right)$ where no specifications are considered on the control inputs for simplicity. In the Boolean point of view, $z[\cdot] \models \Phi$ means that a signal $z[\cdot] \in \mathcal{Z}[\cdot]$ satisfies the specification $\Phi$. Consequently, projection from a Boolean point of view of a specification to a set point of view is possible, where $\Phi=\{z[\cdot] \in \mathcal{Z}[\cdot]: z[\cdot] \models \Phi\}$. It is obvious then that $z[\cdot] \in \Phi$ if and only if $z[\cdot] \models \Phi$. We note that it will be clear from the context whether a specification is interpreted from the set or Boolean point of view.

Next, we define an assume-guarantee contract as given in [3]:

Definition 4: (Assume-Guarantee Contract) An assumeguarantee contract $\mathcal{C}$ is a pair $\left(\Phi_{a}, \Phi_{g}\right)$ consisting of an assumption specification $\Phi_{a}$ and a guarantee specification $\Phi_{g}$ such that $\Phi_{a} \Rightarrow \Phi_{g}$ holds true.

We say that a system $\Sigma$ satisfies $\mathcal{C}=\left(\Phi_{a}, \Phi_{g}\right)$, if $\Sigma \cap \Phi_{a} \subseteq \Phi_{g}$. It can also be easily shown that $\left(\Phi_{a}, \Phi_{g}\right)=$ $\left(\Phi_{a}, \Phi_{a} \Rightarrow \Phi_{g}\right)$. This transformation of the contract is useful when dealing with parametric assume-guarantee contracts for a system $\Sigma\left(\mathcal{U}_{e}[\cdot], \mathcal{U}_{c}[\cdot], \mathcal{U}_{f}[\cdot], \mathcal{Y}[\cdot], \mathcal{K}\right)$, which are given as the following.

Definition 5: (Parametric Assume-Guarantee Contract) An assume-guarantee contract $\mathcal{C}=\left(\Phi_{a}, \Phi_{g}\right)$ is in parametric form if there exists an external parametric assumption specification $\Psi_{a e}: \mathcal{P}_{a e} \mapsto 2^{\mathcal{U}_{e}[\cdot]}$, an internal parametric assumption specification $\Psi_{a f}: \mathcal{P}_{a f} \mapsto 2^{\mathcal{U}_{f}[\cdot]}$, a parametric guarantee specification $\Psi_{g}: \mathcal{P}_{g} \mapsto 2^{\mathcal{Y}[\cdot]}$, and parameter map $\lambda: \mathcal{P}_{a e} \times \mathcal{P}_{a f} \mapsto \mathcal{P}_{g}$ such that:

$$
\Phi_{a}=\bigvee_{\left(p_{e}, p_{f}\right) \in \mathcal{P}_{a e} \times \mathcal{P}_{a f}}\left(\Psi_{a e}\left(p_{e}\right) \wedge \Psi_{a f}\left(p_{f}\right)\right)
$$




$$
\begin{aligned}
\Phi_{g}=\bigwedge_{\left(p_{e}, p_{f}\right) \in \mathcal{P}_{a e} \times \mathcal{P}_{a f}}\left(\left(\Psi_{a e}\left(p_{e}\right)\right.\right. & \left.\wedge \Psi_{a f}\left(p_{f}\right)\right) \\
& \left.\Rightarrow \Psi_{g}\left(\lambda\left(p_{e}, p_{f}\right)\right)\right),
\end{aligned}
$$

where $\mathcal{P}_{a e}, \mathcal{P}_{a f}$, and $\mathcal{P}_{g}$ are the external input parametric set, internal input parametric set, and output parametric set. We note that no specifications over the control input signals are considered.

Remark 1: In case a system does not have an internal input then $\Phi_{a}$ and $\Phi_{g}$ in (4) reduces to:

$$
\begin{gathered}
\Phi_{a}=\bigvee_{p_{e} \in \mathcal{P}_{a e}} \Psi_{a e}\left(p_{e}\right) \\
\Phi_{g}=\bigwedge_{p_{e} \in \mathcal{P}_{a e}}\left(\Psi_{a e}\left(p_{e}\right) \Rightarrow \Psi_{g}\left(\lambda\left(p_{e}\right)\right)\right) .
\end{gathered}
$$

A parametric assume-guarantee contract could be also written as a conjunction of smaller contracts:

$$
\mathcal{C}=\bigwedge_{\left(p_{e}, p_{f}\right) \in \mathcal{P}_{a e} \times \mathcal{P}_{a f}} \mathcal{C}\left(p_{e}, p_{f}\right),
$$

where $\mathcal{C}\left(p_{e}, p_{f}\right)=\left(\Psi_{a e}\left(p_{e}\right) \wedge \Psi_{a f}\left(p_{f}\right),\left(\Psi_{a e}\left(p_{e}\right) \wedge\right.\right.$ $\left.\Psi_{a f}\left(p_{f}\right)\right) \Rightarrow \Psi_{g}\left(\lambda\left(p_{e}, p_{f}\right)\right)$ and it is clear that system $\Sigma$ satisfies $\mathcal{C}$ if for all $\left(p_{e}, p_{f}\right) \in \mathcal{P}_{a e} \times \mathcal{P}_{a f}$, one gets $\Sigma \cap$ $\left(\Psi_{a e}\left(p_{e}\right) \cap \Psi_{a f}\left(p_{f}\right)\right) \subseteq \Psi_{g}\left(\lambda\left(p_{e}, p_{f}\right)\right)$. Definition 5 says that a system under a given parametric AGC must satisfy only the guarantee specifications whose corresponding assumption specifications are triggered. In this paper, we are interested in designing a controller for an interconnected system so that it satisfies a parametric assume-guarantee contract, or equivalently we provide a solution to the following problem:

Problem 1: Consider an interconnection $\mathcal{I}=$ $\left(\Sigma^{1}, \ldots, \Sigma^{N}\right)$ and a set of parametric assumeguarantee contracts $\left\{\mathcal{C}^{1}, \ldots, \mathcal{C}^{N}\right\}$. Design control maps $\mathcal{K}^{i}: \mathcal{Y}^{i}[\cdot] \rightarrow \mathcal{U}_{c}^{i}[\cdot], i \in \mathbb{N}_{[1, N]}$, such that each system $\Sigma^{i}$ satisfies the contract $\mathcal{C}^{i}, i \in \mathbb{N}_{[1, N]}$, and that there exists a global parametric AGC which $\mathcal{I}\left(\Sigma^{1}, \ldots, \Sigma^{N}\right)$ satisfies.

Problem 1 states that we are in search for a decentralized controller that enforces a global parametric $\mathrm{AGC} \mathcal{C}$, to be determined as a function of $\left\{\mathcal{C}^{1}, \ldots, \mathcal{C}^{N}\right\}$, on the behavior of the interconnected system after enforcing local parametric AGCs on its components. In Section III, We derive a generalized small-gain theorem (SGT) for parametric contracts synthesis. In Section V, we recover a classical small-gain result that ensures BIBO stability of an interconnected system.

\section{A General SGT For PARAMETRIC ASSUME-GuARANTEE CONTRACTS}

This section presents the main results in this manuscript by solving Problem 1 . The parametric AGCs $\mathcal{C}^{i}=\left(\Phi_{a}^{i}, \Phi_{g}^{i}\right)$, $i \in \mathbb{N}_{[1, N]}$, are given where $\Phi_{a}^{i}$ and $\Phi_{g}^{i}$ are defined by (4) with specifications $\Psi_{a f}^{i}, \Psi_{a e}^{i}$, and $\Psi_{g}^{i}$ and parameter sets $\mathcal{P}_{a f}^{i}, \mathcal{P}_{a e}^{i}$, and $\mathcal{P}_{g}^{i}$.

Also each subsystem $\Sigma^{i}\left(\mathcal{U}_{e}^{i}[\cdot], \mathcal{U}_{c}^{i}[\cdot], \mathcal{U}_{f}^{i}[\cdot], \mathcal{Y}^{i}[\cdot], \mathcal{K}^{i}\right), i \in$ $\mathbb{N}_{[1, N]}$, has an internal input and an output satisfying:
Assumption 1: The input sets $\mathcal{U}_{f}^{i}[\cdot], i \in \mathbb{N}_{[1, N]}$, are given

$$
\mathcal{U}_{f}^{i}[\cdot]=\mathcal{Y}^{i_{1}}[\cdot] \times \cdots \times \mathcal{Y}^{i_{N-1}}[\cdot] .
$$

where $i_{1}, \ldots, i_{N-1} \in \bar{N}(i)$ and $i_{j} \neq i_{k}$ for $j \neq k$.

Assumption 1 is made for the well-posedness of the interconnection. The internal assumption parameter sets are further partitioned:

Assumption 2: The internal assumption parameter sets $\mathcal{P}_{\text {af }}^{i}, i \in \mathbb{N}_{[1, N]}$, satisfy

$$
\mathcal{P}_{a f}^{i}=\mathcal{P}_{a f}^{i i_{1}} \times \cdots \times \mathcal{P}_{a f}^{i i_{N}},
$$

where $i_{1}, \ldots, i_{N-1} \in \bar{N}(i)$ and $i_{j} \neq i_{k}$ for $j \neq k$.

Following Assumptions 1 and 2, we assume that $\Psi_{a f}^{i}$ is a conjunction of specifications:

Assumption 3: The specifications $\Psi_{a f}^{i}: \mathcal{P}_{a f}^{i i_{1}} \times \cdots \times$

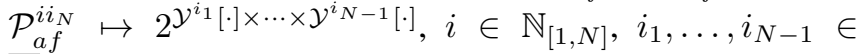
$\bar{N}(i)$ and $i_{j} \neq i_{k}$ for $j \neq k$, satisfy:

$$
\Psi_{a f}^{i}\left(p_{f}^{i}\right)=\bigwedge_{j \in \bar{N}(i)} \Psi_{a f}^{i j}\left(p_{f}^{i j}\right),
$$

where $\Psi_{a f}^{i j}: \mathcal{P}_{a f}^{i j} \mapsto 2^{\mathcal{Y}^{j}}[\cdot]$ and $p_{f}^{i}=\left(p_{f}^{i i_{1}}, \ldots, p_{f}^{i i_{N-1}}\right)$.

Assumptions 2 and 3 restricts the internal parametric assumption specification to be a conjunction of specifications made on the behavior of each subsystem separately. This assumption although not necessary to get a parametric AGC that the interconnected system satisfy, but is needed in Lemma 1 to simplify the obtained guarantee. The parametric guarantee specification for $\Sigma^{i}, i \in \mathbb{N}_{[1, N]}$, is also given by $\Psi_{g}^{i}: \mathcal{P}_{g}^{i} \mapsto 2^{\mathcal{Y}^{i}[\cdot]}$ and the parameter map in Definition 5, associated to system $\Sigma^{i}$, is given by

$$
\lambda^{i}: \mathcal{P}_{a e}^{i} \times \mathcal{P}_{a f}^{i} \mapsto \mathcal{P}_{g}^{i}
$$

One last assumption is made on the assumption and guarantee specifications in order to simplify the guarantee satisfied by the interconnected system. First with respect to each subsystem its own parametric AGC must be satisfied and the guarantees of the other subsystems must imply the former's own assumptions. Second, there must exist at least one external and one internal parameter such that the assumption specifications are satisfied.

Assumption 4: Consider Assumptions 1, 2, 3, $\mathcal{I}\left(\Sigma^{1}, \ldots, \Sigma^{N}\right)$, and a set of parametric assume-guarantee contracts $\left\{\mathcal{C}^{1}, \ldots, \mathcal{C}^{N}\right\}$. Then:

1) There exist control maps $\mathcal{K}^{i}: \mathcal{Y}^{i} \rightarrow \mathcal{U}_{c}^{i}, i \in \mathbb{N}_{[1, N]}$, such that every system $\Sigma^{i}$ satisfies its parametric assume-guarantee contract $\mathcal{C}^{i}=\left(\Phi_{a}^{i}, \Phi_{g}^{i}\right)$.

2) The parameter sets satisfy $\mathcal{P}_{g}^{i_{j}} \subseteq \mathcal{P}_{a f}^{i i_{j}}, j \in \mathbb{N}_{[1, N-1]}$, $i_{j} \in \bar{N}(i)$, and $i \in \mathbb{N}_{[1, N]}$. Also, the guarantee specifications must imply the internal assumption specifications:

$$
\Psi_{g}^{i_{j}}(p) \Rightarrow \Psi_{a f}^{i i_{j}}(p)
$$

for $p \in \mathcal{P}_{g}^{i_{j}}, i_{j} \in \bar{N}(i), j \in \mathbb{N}_{[1, N-1]}$, and $i \in \mathbb{N}_{[1, N]}$. 
3) There exists an external parameter $p_{e}=$ $\left(p_{e}^{1}, \ldots, p_{e}^{N}\right) \in \mathcal{P}_{a e}^{1} \times \cdots \times \mathcal{P}_{a e}^{N}$ such that for all $i \in \mathbb{N}_{[1, N]}, \Psi_{a e}^{i}\left(p_{e}^{i}\right)$ is satisfied.

4) There exists an internal parameter $p_{f}[0]=$ $\left(p_{f}^{1}[0], \ldots, p_{f}^{N}[0]\right) \in \mathcal{P}_{a f}^{1} \times \cdots \times \mathcal{P}_{a f}^{N}$ such that $\Psi_{a f}^{i}\left(p_{f}^{i}\right)$ is satisfied for all $i \in \mathbb{N}_{[1, N]}$.

Remark 2: The first item of Assumption 4 or equivalently verifying whether or not subsystem $\Sigma^{i}$ satisfies the parametric AGC $\mathcal{C}^{i}$ is checked by following the falsification procedure as explained in Section IV.

Before presenting the main result, we state a lemma which will be used in the former's proof.

Lemma 1: Consider Assumptions $1,2,3, \mathcal{I}\left(\Sigma^{1}, \ldots, \Sigma^{N}\right)$, and a set of parametric assume-guarantee contracts $\left\{\mathcal{C}^{1}, \ldots, \mathcal{C}^{N}\right\}$. Suppose that Assumption 4 holds with internal and external parameters $p_{f}[0]$ and $p_{e}$ respectively. In addition, define for every contract $\mathcal{C}^{i}, i \in \mathbb{N}_{[1, N]}$, and using function of $\lambda^{i}$ in (9), a new internal parameter map $\hat{\lambda}^{i}(\cdot)=\lambda^{i}\left(p_{e}^{i}, \cdot\right)$ and define guarantee parameter iterations

$$
p_{g}[k+1]=\Gamma\left(p_{g}[k]\right), \quad k \in \mathbb{N},
$$

with $p_{g}[k]=\left[p_{g}^{1}[k] ; \ldots ; p_{g}^{N}[k]\right], \Gamma=\left[\hat{\lambda}^{1} ; \ldots ; \hat{\lambda}^{N}\right]$, and $p_{g}^{i}[0]=\hat{\lambda}^{i}\left(p_{f}[0], \ldots, p_{f}^{N}[0]\right), i \in \mathbb{N}_{[1, N]}$. Then the guarantee simplifies to

$$
\bigwedge_{i \in \mathbb{N}_{[1, N]}} \bigwedge_{k \in \mathbb{N}} \Psi_{g}^{i}\left(p_{g}^{i}[k]\right)
$$

We note that a similar result to Lemma 1 exists in [20] for the case of subsystems with no control inputs. By exploiting some additional assumptions on the map $\Gamma$ in (11) the guarantee in (12) could be further simplified.

Theorem 1: (SGT for parametric AGCs) Consider $\mathcal{I}\left(\Sigma^{1}, \ldots, \Sigma^{N}\right)$ and a set of parametric assume-guarantee contracts $\left\{\mathcal{C}^{1}, \ldots, \mathcal{C}^{N}\right\}$. Suppose that the assumptions of Lemma 1, with an external parameter $p_{e}=\left(p_{e}^{1}, \ldots, p_{e}^{N}\right)$, hold. Assume also that

1) For every $i \in \mathbb{N}_{[1, N]}$ there exists a metric $\mathbf{d}^{\mathbf{i}}: \mathcal{P}_{g}^{i} \times$ $\mathcal{P}_{g}^{i} \mapsto \mathbb{R}_{0}^{+}$on $\mathcal{P}_{g}^{i}$. The Hausdorff distance $\mathbf{d}_{\mathbf{H}}$ is also a metric on $\Psi_{g}^{i}(\cdot), i \in \mathbb{N}_{[1, N]}$.

2) The specification $\Psi_{g}^{i}$ varies continuously with parameters in $\mathcal{P}_{g}^{i}, i \in \mathbb{N}_{[1, N]}$. In other words, for every $\varepsilon^{i}>0$ and $p_{1} \in \mathcal{P}_{g}^{i}$ there exists a $\delta^{i}>0$ such that

$$
\mathbf{d}^{\mathbf{i}}\left(p_{1}, p\right)<\delta^{i} \Rightarrow \mathbf{d}_{\mathbf{H}}\left(\Psi_{g}^{i}\left(p_{1}\right), \Psi_{g}^{i}(p)\right)<\varepsilon^{i},
$$

for $i \in \mathbb{N}_{[1, N]}$.

3) The sequence $\left(p_{g}[k]\right)_{k \in \mathbb{N}}$ satisfying (11) converges for any $p_{g}[0] \in \mathcal{P}_{g}^{1} \times \cdots \times \mathcal{P}_{g}^{N}$ to a parameter $\hat{p}_{g}=$ $\left[\hat{p}_{g}^{1} ; \ldots ; \hat{p}_{g}^{N}\right]$.

Then $\mathcal{I}\left(\Sigma^{1}, \ldots, \Sigma^{N}\right)$ satisfies the parametric AGC $\mathcal{C}=$ $\left(\Phi_{a}, \Phi_{g}\right)$ given by (5) with $\mathcal{P}_{a e}=\prod_{i=1}^{N} \mathcal{P}_{a e}^{i}, \mathcal{P}_{g}=\prod_{i=1}^{N} \mathcal{P}_{g}^{i}$, $\Psi_{a e}\left(p_{e}\right)=\bigwedge_{i \in \mathbb{N}_{[1, N]}} \Psi_{a e}^{i}\left(p_{e}^{i}\right), \lambda\left(p_{e}\right)=\hat{p}_{g}$, and

$$
\Psi_{g}\left(\hat{p}_{g}\right)=\bigwedge_{i \in \mathbb{N}_{[1, N]}}\left(\mathbf{c l}\left(\Psi_{g}^{i}\left(\hat{p}_{g}^{i}\right)\right)\right) .
$$

\section{CONTROLLER SYNTHESIS FOR PARAMETRIC ASSUME-GUARANTEE CONTRACTS}

In this section, we design a control map $\mathcal{K}^{i}: \mathcal{Y}^{i} \rightarrow \mathcal{U}_{c}^{i}$ for a single subsystem $\Sigma^{i}, i \in \mathbb{N}_{[1, N]}$, so that it satisfies a given parametric AGC $\mathcal{C}^{i}$. For the sake of better readability we drop the index $i$ and we assume that $\mathcal{C}$ has a fixed external input parameter. The overall method is summarized in Algorithm 1.

Line 3 corresponds to the partitioning of the internal assumption parameter set $\mathcal{P}_{a f}$ into $L$ sets $\left\{\mathcal{S}_{k}\right\}_{k \in \mathbb{N}_{[1, L]}}$ with $\left\{s_{k}\right\}_{k \in \mathbb{N}_{[1, L]}}$ being some fixed parameters such that $s_{k} \in \mathcal{S}_{k}$, $k \in \mathbb{N}_{[1, L]}$. Line 4 synthesizes for $k \in \mathbb{N}_{[1, L]}$ a controller $K_{k}$ so that $\Sigma$ satisfies the parametric AGC $\mathcal{C}$ when fixing the parameter to $s_{k}$. Line 6 , and in case the synthesis of all controllers $K_{k}$ was successful, verifies whether or not for $k \in \mathbb{N}_{[1, L]}$, system $\Sigma$ satisfies the contract $\mathcal{C}$ with a controller $K_{k}$ for all internal assumption parameters that belong to the set $\mathcal{S}$. Note that in this step verification is checked by following the falsification procedure as explained in [3]. Lines $9-10$, and if verification is successful for all controllers $K_{k}, k \in \mathbb{N}_{[1, L]}$, returns a control map $\mathcal{K}$. Practically, $\mathcal{K}$ checks online in which region $\mathcal{S}_{k}, k \in \mathbb{N}_{[1, L]}$, the internal assumption parameter belongs to and then enforces the guarantees of the parametric AGC $\mathcal{C}$ via $K_{k}$. In case the synthesis of the control map was not successful, set $\mathcal{P}_{a f}$ is further partitioned into finer sets until reaching a granularity corresponding to $L_{\max }$ sets. In that case, and if no control map is designed then Algorithm 1 returns unknown indicating that our method fails in synthesizing a control map for $\Sigma$.

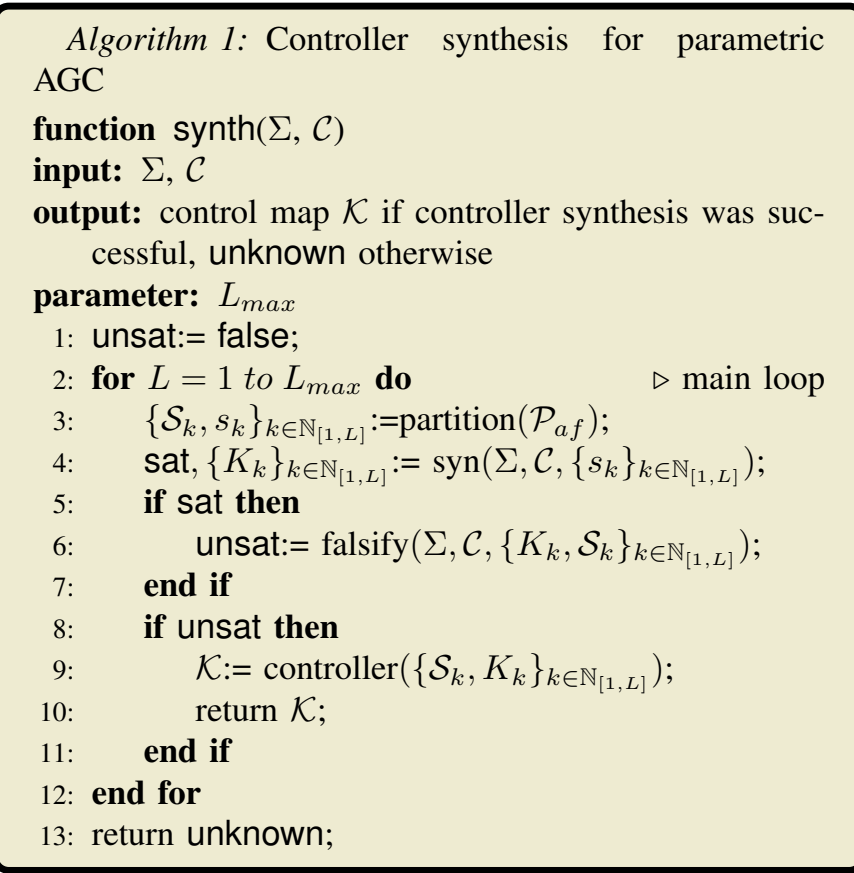

\section{SGT ON Bounded InPut Bounded OUtPut STABILITY}

Using the proposed results in the previous sections and under an additional assumption on the map $\Gamma$ in (11), we 
recover here the classical small-gain theorem on bounded input bounded output (BIBO) stability of an interconnected system which is analogous to the asymptotic gain property (AG) in [6]. In this section, we assume that we have already designed controllers for subsystems that makes them BIBO stable (i.e. each subsystem satisfies (15)) using existing methods [22]. Then we consider for simplicity dynamical systems with no control inputs, i.e. $\Sigma\left(\mathcal{U}_{e}[\cdot], \mathcal{U}_{f}[\cdot], \mathcal{Y}[\cdot]\right)$.

Given a norm $|\cdot|$, we denote by $\mathcal{L}$ the set of norm bounded signals. A function $\gamma: \mathbb{R}_{0}^{+} \mapsto \mathbb{R}^{+}$is said to be of class $\mathcal{K}$, or $\gamma \in \mathcal{K}$, if it is continuous, increasing, and $\gamma(0)=0$. We say $\gamma \in \mathcal{K}_{\infty}$ if it is of class $\mathcal{K}$ and unbounded. For later derivations, we define a map $\Gamma_{s}:\left(\mathbb{R}^{+}\right)^{N} \mapsto\left(\mathbb{R}^{+}\right)^{N}$, which is used in Corollary 1 to identify the map $\Gamma$.

Definition 6: $\Gamma_{s}:\left(\mathbb{R}_{0}^{+}\right)^{N} \mapsto\left(\mathbb{R}_{0}^{+}\right)^{N}$ is an irreducible map satisfying

$$
\left(\Gamma_{s} \circ D_{s}\right)(s) \not s, \quad \forall s \in\left(\mathbb{R}^{+}\right)^{N},
$$

where $D:\left(\mathbb{R}_{0}^{+}\right)^{N} \mapsto\left(\mathbb{R}_{0}^{+}\right)^{N}$ is defined by $D_{s}\left(s^{1}, \ldots, s^{N}\right)=\left[\left(I d+\alpha^{1}\right)\left(s^{1}\right) ; \ldots ;\left(I d+\alpha^{N}\right)\left(s^{N}\right)\right]$, for some $\alpha^{i} \in \mathcal{K}_{\infty}, i \in \mathbb{N}_{[1, N]}$.

The next result follows from Theorem 1.

Corollary 1: (SGT on BIBO stability) Consider an interconnected system $\mathcal{I}\left(\Sigma^{1}, \ldots, \Sigma^{N}\right)$, with $\Sigma^{i}\left(\mathcal{L}, \mathcal{L}^{N-1}, \mathcal{L}\right)$, $i \in \mathbb{N}_{[1, N]}$. Assume that for every $i \in \mathbb{N}_{[1, N]}$, there exist $\gamma^{i 1}, \ldots, \gamma^{i N}, \gamma^{u_{i}} \in \mathcal{K} \cup\{0\}, \gamma^{i i}=0$, such that

$$
\left|y^{i}[\cdot]\right| \leq \sum_{j \in \mathbb{N}} \gamma^{i j}\left(\left|y^{j}[\cdot]\right|\right)+\gamma^{u_{i}}\left(\left|u^{i}[\cdot]\right|\right) .
$$

If $\Gamma_{s}:\left(\mathbb{R}_{0}^{+}\right)^{N} \mapsto\left(\mathbb{R}_{0}^{+}\right)^{N}$, defined by

$$
\Gamma_{s}\left(s_{1}, \ldots, s_{N}\right)=\left[\sum_{j=1}^{N} \gamma^{1 j}\left(s_{j}\right) ; \ldots ; \sum_{j=1}^{N} \gamma^{n N}\left(s_{j}\right)\right],
$$

satisfies Assumption 6, then there exists a $\beta \in \mathcal{K}_{\infty}$ such that:

$$
\left.|| y[\cdot]\right|_{v} \mid \leq \beta\left(\left|D\left(\left.|u[\cdot]|\right|_{v}\right)\right|\right)
$$

where $|y[\cdot]|_{v}=\left[\left|y^{1}[\cdot]\right| ; \ldots ;\left|y^{N}[\cdot]\right|\right], \quad D=$ $\operatorname{diag}\left(\gamma_{1}^{u}, \ldots, \gamma_{N}^{u}\right)$, and $|u[\cdot]|_{v}=\left[\left|u^{1}[\cdot]\right| ; \ldots ;\left|u^{N}[\cdot]\right|\right]$.

In the next section we present a large-scale transportation system to demonstrate the results obtained by Theorem 1 .

\section{ILLUSTRATIVE EXAMPLE}

We consider a large-scale transportation system $\mathcal{I}\left(\Sigma^{1}, \ldots, \Sigma^{N}\right)$ consisting of $N \in \mathbb{N}_{[3,+\infty)}$ interconnected segments $\Sigma^{i}$, depicted each by Figure 2 . Subsystem $\Sigma^{i}$

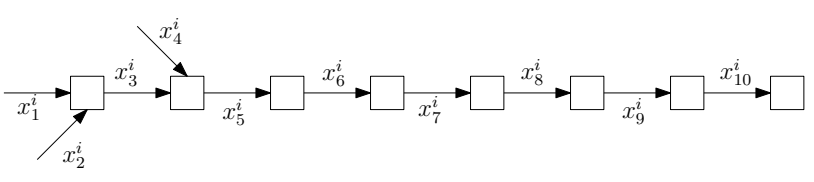

Fig. 2: Model of subsystem $\Sigma^{i}$. consists of 10 links $l_{1}^{i}, \ldots, l_{10}^{i}$ and is given by the discrete dynamics, as in [23]:

$$
\begin{aligned}
x_{1}^{i}[k+1] & =x_{1}^{i}[k]-f_{1}^{\text {out }_{i}}[k]+f_{1}^{\text {in }_{i}}[k] \\
x_{2}^{i}[k+1] & =x_{2}^{i}[k]-f_{2}^{\text {out }_{i}}[k]+f_{2}^{\text {in }_{i}}[k] \\
x_{3}^{i}[k+1] & =x_{3}^{i}[k]-f_{3}^{\text {out }_{i}}[k]+f_{1}^{\text {out }_{i}}[k]+f_{2}^{\text {out }_{i}}[k] \\
x_{4}^{i}[k+1] & =x_{4}^{i}[k]-f_{4}^{\text {out }_{i}}[k]+f_{4}^{\text {in }_{i}}[k] \\
x_{5}^{i}[k+1] & =x_{5}^{i}[k]-f_{5}^{\text {out }_{i}}[k]+f_{3}^{\text {out }_{i}}[k]+f_{4}^{\text {out }_{i}}[k] \\
x_{j}^{i}[k+1] & =x_{j}^{i}[k]-f_{j}^{\text {out }_{i}}[k]+f_{j-1}^{\text {out }_{i}}[k], \quad j \in \mathbb{N}_{[6,10]}
\end{aligned}
$$

where $x_{j}^{i} \in \mathbb{R}_{0}^{+}$represents the average number of vehicles in link $l_{j}^{i}, j \in \mathbb{N}_{[1,10]}$ and the output of $\Sigma^{i}$ is $\left[x_{1}^{i}[\cdot] ; x_{10}^{i}[\cdot]\right]$.

The interconnection $\mathcal{I}$ is given by (2) with $\mathcal{G} \in$ $\mathbb{R}^{2 N(N-1) \times 2 N}$ defined in accordance with Figure 3 .

The flows out of links $l_{1}^{i}, \ldots, l_{9}^{i}$ are given, for $i \in \mathbb{N}_{[1, N]}$, by:

$$
\begin{aligned}
& f_{1}^{\text {out }_{i}}[k]=\min \left(0.8\left(40-x_{3}^{i}[k]\right), 10, x_{1}^{i}[k]\right), \\
& f_{2}^{\text {out }_{i}}[k]=\min \left(0.2\left(40-x_{3}^{i}[k]\right), 5, x_{2}^{i}[k]\right), \\
& f_{3}^{\text {out }_{i}}[k]=\min \left(0.8\left(40-x_{5}^{i}[k]\right), 10, x_{3}^{i}[k]\right), \\
& f_{4}^{\text {out }_{i}}[k]=\min \left(0.2\left(40-x_{5}^{i}[k]\right), 5, x_{4}^{i}[k]\right), \\
& f_{j}^{\text {out }_{i}}[k]=\min \left(0.8\left(40-x_{j+1}^{i}[k]\right), 10, x_{j}^{i}[k]\right), j \in \mathbb{N}_{[5,9]},
\end{aligned}
$$

As for links $l_{10}^{i}, i \in \mathbb{N}_{[1, N]}$, the output flows are given by:

$f_{10}^{\text {out }_{i}}[k]=\min \left(0.2\left(40-x_{1}^{i+1}[k]\right), 10 u^{i}, x_{10}^{i}[k]\right), i \in \mathbb{N}_{[1, N-1]}$, $f_{10}^{\text {out }_{N}}[k]=\min \left(0.2\left(40-x_{1}^{1}[k]\right), 10 u^{N}, x_{10}^{N}[k]\right)$,

where $u^{i} \in[0,1], i \in \mathbb{N}_{[1, N]}$, are control inputs, representing ramp meters, to be determined. Furthermore, the flows into links are given by:

$$
\begin{aligned}
f_{1}^{i n_{1}}[k] & =f_{10}^{\text {out }_{N}}[k], \\
f_{1}^{i n_{i}}[k] & =\min \left(0.8\left(40-x_{1}^{i}[k]\right), 10, x_{10}^{i-1}[k]\right), i \in \mathbb{N}_{[2, N]}, \\
f_{2}^{i n_{i}}[k] & =\min \left(\left(20-x_{2}^{i}[k]\right), d_{2}^{i}[k]\right), i \in \mathbb{N}_{[1, N]}, \\
f_{4}^{i n_{i}}[k] & =\min \left(\left(20-x_{4}^{i}[k]\right), d_{4}^{i}[k]\right), i \in \mathbb{N}_{[1, N]} .
\end{aligned}
$$

\section{A. Controller synthesis for certifying assume-guarantee con-} tracts

For all subsystems, the on-ramp demands are limited to be always less than 3 :

$$
\square\left(d_{j}^{i} \leq 3\right), j \in\{2,4\}, i \in \mathbb{N}_{[1, N]}
$$

All links are assumed to have an initial number of vehicles less than 4. Using signal temporal logic (STL) formulas [24], we consider the parametric assume-guarantee contracts $\mathcal{C}^{i}=$

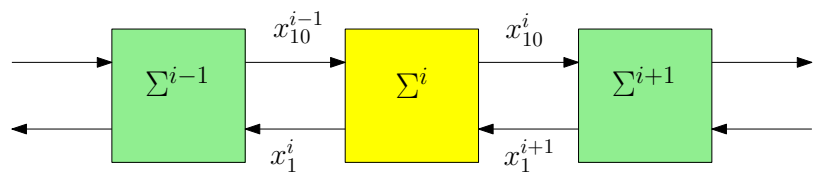

Fig. 3: Interconnections of subsystem $\Sigma^{i} i \notin\{1, N\}$. 
$\left(\Phi_{a}^{i}, \Phi_{g}^{i}\right), i \in \mathbb{N}_{[1, N]}$ with:

$$
\begin{aligned}
& \Phi_{a}^{i}=\bigvee_{s^{i} \geq 0, d^{i} \geq 0}\left(\square_{[0,3]} \nabla_{[0,2]}\left(s\left(x_{1}^{i+1}\right) \geq 10-s^{i}\right)\right. \\
& \left.\wedge \square_{[0,3]} \diamond_{[0,2]}\left(\min \left(10, x_{10}^{i-1}\right) \leq d^{i}\right)\right) \\
& \Phi_{g}^{i}=\bigwedge_{s^{i} \geq 0, d^{i} \geq 0}\left(\left(\square_{[0,3]} \triangleright_{[0,2]}\left(s\left(x_{1}^{i+1}\right) \geq 10-s^{i}\right)\right.\right. \\
& \left.\wedge \square_{[0,3]} \diamond_{[0,2]}\left(\min \left(10, x_{10}^{i-1}\right) \leq d^{i}\right)\right) \\
& \Rightarrow\left(\square_{[0,3]} \diamond_{[0,2]}\left(s\left(x_{1}^{i}\right) \geq 10-\lambda_{2}\left(d^{i}\right)\right)\right. \\
& \left.\left.\wedge \square_{[0,3]} \oslash_{[0,2]}\left(\min \left(10, x_{10}^{i}\right) \leq \lambda_{1}\left(s^{i}\right)\right)\right)\right)
\end{aligned}
$$

for $i \in \mathbb{N}_{[2, N-1]}$, where $s(x)=0.8(40-x), \lambda_{1}(s)=$ $0.9 s+6.1$, and $\lambda_{2}(d)=0.2 d$. We note that the assumption and guarantee specifications for $\mathcal{C}^{1}$ and $\mathcal{C}^{N}$ are similar to (19) but for $\mathcal{C}^{1}$ the assumptions are made on the states $x_{1}^{2}$ and $x_{10}^{N}$ whereas for $\mathcal{C}^{N}$ the assumptions are made on $x_{1}^{1}$ and $x_{10}^{N-1}$. Following Remark 2 and Algorithm 1, we reformulate the controller synthesis problem, for each modular component, having signal temporal logic formulas into mixed integer linear programs [25]. Using the Gurobi optimization tool [26] the latter problem was solved successfully and a control map $\mathcal{K}^{i}$ that defines the control inputs $u^{i}, i \in \mathbb{N}_{[1, N]}$, is synthesized. Therefore $\Sigma^{i}$ with $\mathcal{K}^{i}$ satisfies the parametric assume-guarantee contract $\left(\phi_{a}^{i}, \phi_{g}^{i}\right), i \in \mathbb{N}_{[1, N]}$. The conditions for the small-gain theorem (i.e. Theorem 1) hold as the following:

- The parametric contracts are satisfied for each network.

- The internal assumptions of any network are implied by guarantees from neighboring networks because they are of the same form.

- The external assumptions are satisfied via (18).

- For any $i \in \mathbb{N}_{[1, N]}$ for a large enough $d^{i} \geq 0$ and $s^{i} \geq 0$, the internal assumption $\Phi_{a}^{i}$ is satisfied because $\min \left(10, x_{10}^{i-1}\right)$ has a maximum value of 10 and $s(x)$ has a minimum value of 0 .

It can also be shown that the sequence of parameters in (11) converges to a limit point $\left[d^{i} ; s^{i}\right]=[7.44 ; 1.48]$, $i \in \mathbb{N}_{[1, N]}$. Thus we conclude that the interconnected system $\mathcal{I}\left(\Sigma^{1}, \ldots, \Sigma^{N}\right)$ is guaranteed to satisfy the following specification, for $i \in \mathbb{N}_{[1, N]}$ :

$$
\begin{gathered}
\square_{[0,3]} \searrow_{[0,2]}\left(s\left(x_{1}^{i}\right) \geq 8.52\right) \wedge \square_{[0,3]} \searrow_{[0,2]}\left(\min \left(10, x_{10}^{i}\right) \leq 7.44\right) . \\
\text { VII. ConCLUSION }
\end{gathered}
$$

ISmall-gain conditions were derived for parametric AGCs allowing for the compositional analysis of a large-scale system based on a fragment of STL specifications. Using these conditions we recovered a classical small-gain theorem guaranteeing BIBO stability for an interconnected system. Also, the validity of our approach was illustrated by a large-scale transportation system. Further investigations are carried to solve the controller synthesis problem in order to enforce a larger class of temporal logic specifications on an interconnected system.

\section{REFERENCES}

[1] C. S. Păsăreanu, D. Giannakopoulou, M. G. Bobaru, J. M. Cobleigh, and $\mathrm{H}$. Barringer, "Learning to divide and conquer: applying the $1^{*}$ algorithm to automate assume-guarantee reasoning," Formal Methods in System Design, vol. 32, no. 3, pp. 175-205, 2008.

[2] A. Benveniste, B. Caillaud, A. Ferrari, L. Mangeruca, R. Passerone, and C. Sofronis, "Multiple viewpoint contract-based specification and design," in International Symposium on Formal Methods for Components and Objects. Springer, 2007, pp. 200-225.

[3] E. Kim, M. Arcak, and S. Seshia, "A small gain theorem for parametric assume-guarantee contracts," in Proceedings of the 20th International Conference on Hybrid Systems: Computation and Control, 2017.

[4] A. Saoud, A. Girard, and L. Fribourg, "Contract based design of symbolic controllers for interconnected multiperiodic sampled-data systems," in Conference on Decision and Control. IEEE, 2018.

[5] E. Kim, M. Arcak, and S. Seshia, "Directed specifications and assumption mining for monotone dynamical systems," in Proceedings of the Conference on Hybrid Systems: Computation and Control, 2016.

[6] S. Dashkovskiy, B. Rüffer, and F. Wirth, "An iss small gain theorem for general networks," Mathematics of Control, Signals, and Systems, vol. 19, no. 2, pp. 93-122, 2007.

[7] A. R. Teel, "A nonlinear small gain theorem for the analysis of control systems with saturation," IEEE transactions on Automatic Control, vol. 41, no. 9, pp. 1256-1270, 1996.

[8] Z.-P. Jiang and Y. Wang, "Input-to-state stability for discrete-time nonlinear systems," Automatica, vol. 37, no. 6, pp. 857-869, 2001.

[9] A. Pnueli, "The temporal logic of programs," in 18th Annual Symposium on Foundations of Computer Science. IEEE, 1977, pp. 46-57.

[10] M. Rungger and M. Zamani, "Compositional construction of approximate abstractions of interconnected control systems," IEEE Transactions on Control of Network Systems, vol. 5, no. 1, pp. 116-127.

[11] P. Tabuada, G. Pappas, and P. Lima, "Compositional abstractions of hybrid control systems," Discrete event dynamic systems, vol. 14, no. 2, pp. 203-238, 2004.

[12] G. Pola, P. Pepe, and M. Di Benedetto, "Symbolic models for networks of control systems," IEEE Transactions on Automatic Control, vol. 61, no. 11, pp. 3663-3668, 2016.

[13] Y. Tazaki and J. Imura, "Bisimilar finite abstractions of interconnected systems," in International Workshop on Hybrid Systems: Computation and Control. Springer, 2008, pp. 514-527.

[14] D. Boskos and D. V. Dimarogonas, "Decentralized abstractions for feedback interconnected multi-agent systems," in 2015 54th IEEE Conference on Decision and Control. IEEE, 2015, pp. 282-287.

[15] P.-J. Meyer, A. Girard, and E. Witrant, "Compositional abstraction and safety synthesis using overlapping symbolic models," IEEE Transactions on Automatic Control, vol. 63, no. 6, pp. 1835-1841, 2018.

[16] E. Dallal and P. Tabuada, "On compositional symbolic controller synthesis inspired by small-gain theorems," in 2015 54th IEEE Conference on Decision and Control (CDC). IEEE, 2015, pp. 6133-6138.

[17] A. Le Coënt, L. Fribourg, N. Markey, F. De Vuyst, and L. Chamoin, "Distributed synthesis of state-dependent switching control," in International Workshop on Reachability Problems. Springer, 2016.

[18] M. Al Khatib, A. Girard, and T. Dang, "Stability verification and timing contract synthesis for linear impulsive systems using reachability analysis," Nonlinear Analysis: Hybrid Systems, vol. 25, 2017.

[19] S. Sadraddini, J. Rudan, and C. Belta, "Formal synthesis of distributed optimal traffic control policies," in 2017 ACM/IEEE 8th International Conference on Cyber-Physical Systems. IEEE, 2017, pp. 15-24.

[20] Y. Chen, J. Anderson, K. Kalsi, S. H. Low, and A. D. Ames, "Compositional set invariance in network systems with assume-guarantee contracts," in 2019 American Control Conference. IEEE, 2019.

[21] E. Kim, M. Arcak, and M. Zamani, "Constructing control system abstractions from modular components," in Proceedings of the 21st International Conference on Hybrid Systems: Computation and Control (part of CPS Week). ACM, 2018, pp. 137-146.

[22] H. K. Khalil, "Nonlinear systems."

[23] C. Daganzo, "The cell transmission model: A dynamic representation of highway traffic consistent with the hydrodynamic theory," Transportation Research Part B: Methodological, vol. 28, pp. 269-287.

[24] V. Raman, A. Donzé, D. Sadigh, R. Murray, and S. Seshia, "Reactive synthesis from signal temporal logic specifications," in Proceedings of the conference on hybrid systems: Computation and control, 2015.

[25] W. Heemels, B. De Schutter, and A. Bemporad, "Equivalence of hybrid dynamical models," Automatica, vol. 37, no. 7, pp. 1085-1091, 2001.

[26] G. OPTIMIZATION, "Inc. gurobi optimizer reference manual," 2014. 